\title{
Diabetes Mellitus as One of the Risk Factors Contribute to Carcinogenesis
}

\author{
Noorwati Sutandyo \\ Department of Hematology-Medical Oncology, Dharmais Cancer Hospital - National Cancer Center, Jakarta, Indonesia
}

\section{ARTICLE INFO}

Received : 01 January 2020

Reviewed : 19 February 2020

Accepted : 20 May 2020

Keywords:

cancer, carcinogenesis, diabetes,

insulin resistance

*Corresponding author:

Noorwati Sutandyo

Department of Hematology-Medical

Oncology, Dharmais Cancer Hospital-

National Cancer Center, Jl Letjen S Parman,

Kav. 84-86, Slipi, Jakarta Barat 11420

noorwatisoetandyo@gmail.com

\author{
A BSTRACT
}

\begin{abstract}
Diabetes mellitus (DM) and cancer are both global diseases whose numbers continue to grow. Higher risk of developing cancer in diabetic patients, especially in liver, endometrial, pancreatic, kidney, colorectal, bladder, and breast cancer, was already shown in several previous studies. This review will explain the possible DM pathogenesis that plays a role in carcinogenesis using the simple but thorough concept of insulin resistance, hyperglycemia, and chronic inflammation. By knowing the link, it is hoped that this review can be useful in developing cancer prevention plans for those with diabetes.
\end{abstract}

\section{INTRODUCTION}

Diabetes mellitus (DM) and cancer are diseases that have experienced a rapid increase throughout the world. In 2017, the number of DM cases was 451 million and is expected to reach 693 million by 2045 [1]. The number of deaths in 2016 was estimated at around 1.6 million and almost half of the cases occurring before the age of 70 years [2]. Meanwhile, cancer death in developing countries continues to increase, with the number of new cases estimated to reach 19.3 million cases per year by 2025 [3].

Diabetes mellitus can be caused by a congenital deficiency or obtained due to reduced insulin production by the pancreas, or by the ineffectiveness of insulin production that progress chronically. The relationship between DM and cancer is shown in diabetic patients who have increased risk of cancer (relative risk of 2.02.5 for liver, endometrial, and pancreatic cancer, and 1.2-1.5 for breast, colon, and bladder cancer) [4].

\section{PATHOGENESIS}

The pathogenesis of DM and cancer is mediated by three main pathways, namely insulin resistance, hyperglycemia, and chronic inflammation. These pathways can have a direct or interconnected effect, which ultimately cones in the process of cancer development (Figure 1).

\section{Insulin Resistance}

Genetic and environmental factors share their roles in insulin resistance. Genetic factors are related to alteration of insulin receptor signaling pathway (INSR) and insulinlike growth factor receptor (IGFR), while environmental factors are related to the sedentary lifestyle and overeating habits associated with obesity [5].

The action of insulin is related to the signal transduction pathway involving insulin receptors and various signal cascades. In normal conditions, insulin interacts with INSR, an integral membrane glycoprotein receptor with intrinsic enzymatic activity. After insulin is bound, the receptor is phosphorylated and activates the insulin receptor substrate (IRS), triggering an intracellular cascade. IRS-1 and IRS-2 are the most common substrates and intermediates in the early stages of insulin signal propagation that regulates molecular complexes and triggers an intracellular signaling cascade. Among the various PI3K-activated kinase pathways, the Akt pathway has a vital role in the metabolic function of insulin. Akt participates in the translocation of glucose-4 (GLUT-4) transport from intracellular vesicles 
to the cell surface to increase glucose absorption. Insulin also participates in the inhibition of gluconeogenesis and supports the synthesis of glycogen, lipids, and proteins in the liver, adipose, and muscle tissue [6]. This pathway is called the PI3K/Akt pathway.

In addition to this pathway, INSR can be potentially oncogenic through the stimulation of abnormal cellular cascades that activate Ras/MAPK pathway. This pathway then activates cell proliferation internally, which depends on growth hormone, and directly influences cell metabolism [7].

Chronic hyperinsulinemia in insulin-resistant conditions also increases the bioavailability of IGF-I as a result of reduced hepatic gene expression and decreases the production of insulin-like growth factor binding proteins (IGFBP). IGF-1 can bind to IGFR and trigger mitogenic and anti-apoptotic effects, through the PI3K/Akt and Ras/MAPK pathways [8]. Several studies have shown that higher IGF-1 levels are associated with an increased risk of cancer incidence and deaths [6].

Insulin-like growth factor binding proteins (IGFBP)-3 is the main binding protein expressed in serum. Most of the circulating IGF-I and IGF-II are bound in a complex with IGFBP-3. Free IGF-I is available biologically to bind to IGF-I receptors (IGF-IR). The binding of IGF-I and IGF-II to IGF-IR inhibits apoptosis and increases cell proliferation. IGF-I can also bind and form heterodimers with insulin receptor subtypes $A$ and $B$ (IR-A and IR-B). It was found that IR-A has a high affinity for insulin and IGF-II but binds IGF-I with low affinity [8].

Liver is the primary location for IGFBP-3 production, and its expression is regulated by growth hormone $(\mathrm{GH})$ and suppressed by insulin [6]. Like IGFBP-3, IGF-I biosynthesis occurs mainly in the liver and also depends on $\mathrm{GH}$ levels, but increases in the presence of insulin. Consistently, higher $\mathrm{GH}$ receptor expression and increased IGF-I protein production can be detected in patients with ongoing hyperinsulinemia and chronic type-2 diabetes [9]. Besides, decreased IGFBP expression in the malignant transformation was reported in several types of tumor cells where the number of free IGF-I increased or even at the rate of IGF-I production remained $[5,7]$.

Insulin and IGF-I inhibit the synthesis of liver hormone-sex-binding globulin (SHBG), resulting in a decrease in circulating levels of SHBG, which contributes to the increase in the estradiol and testosterone bioavailability. This inhibition harms target cells and tissues that express estrogen and androgen receptors. The binding effect of sex steroids with specific receptors varies but can produce abnormal cell proliferation and inhibition of apoptosis in some tissues, specifically the breast epithelium and endometrium [8].

In obesity, insulin resistance is associated with hypoxic conditions in adipose tissue. Fatty cells that are over-expanded can become hypoxic and activate the hypoxia-inducible factor 1 (HIF-1) gene. This process leads to increased expression of JNK and IKK and creates an inflammatory environment in fat cells that can cause insulin resistance [10].

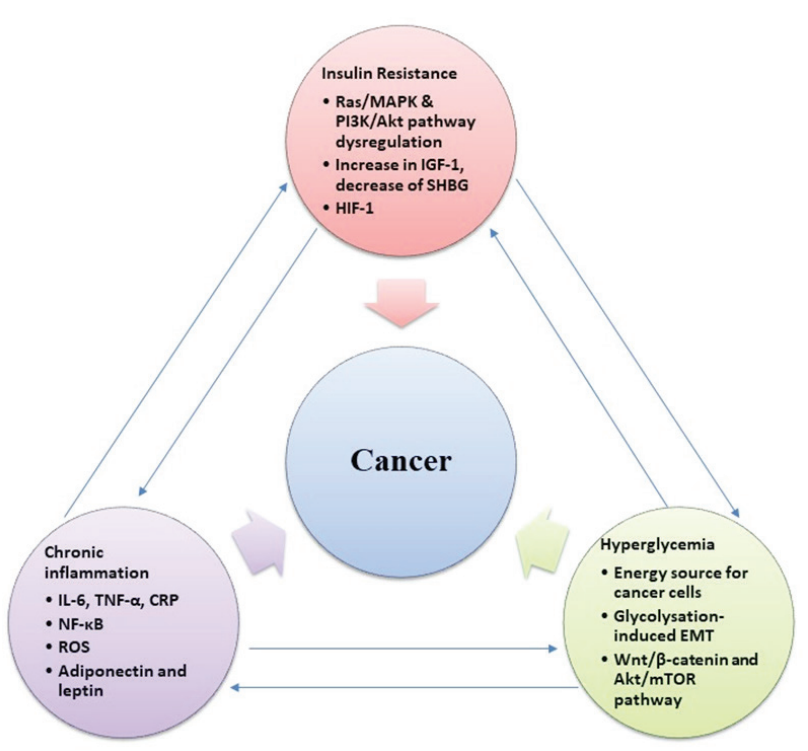

INSR, insulin receptor gene; IGF-I, insulin-like growth factor I; HIF-1, hypoxiainducible factor; TNF- $\alpha$, tumor necrosis factor $\alpha$; IL-6, interleukin-6; SHBG, sex-hormone-binding globulin; ROS, reactive oxygen species; NF-KB, nuclear factor-kappa B; EMT, epithelial-mesenchymal transition; MAPK, mitogen activating protein kinase; PI3K, Phosphoinositide 3-kinases; mTOR, mammalian target of rapamycin.

Figure 1. A simple scheme of the relationship between DM pathogenesis and cancer

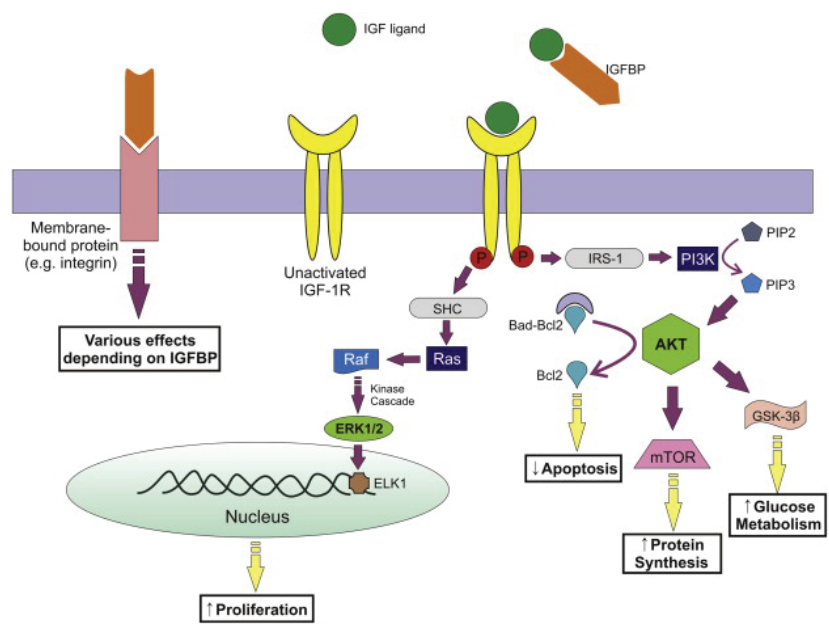

Figure 2. Schematic of IGF signaling on IGF-1R signaling cascade. Activation of IGF-1R can result in signaling via two pathways: PI3K/AKT and Ras/MAPK7 [7]

\section{Hyperglycemia}

Possible pathogenesis of hyperglycemia in increasing the risk of cancer can be divided into two, namely indirect and direct effects. An indirect effect is called when the initial effects of hyperglycemia occur in other organs, which then induces IGF-1 and inflammatory cytokines that trigger tumor cell growth. The direct 
effect of hyperglycemia works on tumor cells by increasing proliferation, inducing mutations, increasing invasion and migration, and improving cancer-related signaling pathways. These two effects work together to induce tumorigenesis in healthy cells [11].

Excess glucose supports the increasing energy and metabolic requirements of cancer cells [11]. Most malignant tissues experience an increase in fludeoxyglucose uptake, associated with an increase in the rate of glycolysis and glucose transport [12]. The glycolytic rate produces various intermediates that can be used as nucleotide biosynthesis, nucleotides, NADPH, lipids, amino acids, and glycoconjugates $[11,12]$.

Two to five percent of glucose entering will experience the hexosamine biosynthetic pathway (HBP) using the enzyme glutamine-fructose-6-phosphate amidotransferase (GFAT) which produces various end products: UDP-GIcNAc and its derivatives (UDP-GaINAc, UDP-ManNAc, and CMP -Neu5Ac). These products can glycosylate proteins pathologically, thus disrupting the function of various organs and tissues $[6,8]$, including between tumor invasion and metastasis, through a process called "epithelial-mesenchymal transition" (EMT) [13].

Besides, hyperglycemia is also associated with an increase in pro-survival Akt/mTOR signaling and WNT/ $\beta$-catenin, which play an essential role in cell proliferation and survival [14]. Disorders of WNT signaling are found in $40-90 \%$ of gastrointestinal cancers, which are the sites of cancer that are strictly related to diabetes [15]. the Chi-square test with confidence interval of $95 \%$.

\section{Chronic Inflammation and Oxidative Stress}

Uncontrolled diabetes results in a proinflammatory process characterized by elevated levels of interleukin- 6 (IL-6), tumor necrosis factor-alpha (TNF- $\alpha$ ), C-reactive protein, and other markers of chronic inflammation. Persistent inflammation increases genetic instability associated with an increased risk of cancer $[16,17]$.

High glucose levels are also associated with an increase in oxidative stress that is formed from metabolic products (mainly from the oxidative phosphorylation in the mitochondria) and the formation of advanced glycation end products (AGE). Oxidative stress can cause damage to lipids, proteins, and DNA that will start or simplify the process of carcinogenesis. Hydrogen peroxide is one of ROS which can act as a second messenger in cellular signaling that regulates the activity of tyrosine phosphatase proteins, tyrosine kinase proteins, tyrosine kinase receptors, and transcription factors. Pathways that can be dysregulated include MAPK/Erk cascade, PI3K/Akt, and IKB kinase (IKK)/K-B factor (NF-KB) activation pathway [18].

Under normal conditions, when inactive, NF-KB is tightly bound to its inhibitor, $\mathrm{KB}$, which is a transcription factor in the cytosol. The presence of cytokine stimulation such as TNFa or IL-1 and $\mathrm{H} 2 \mathrm{O} 2$ (hydrogen peroxide) levels can activate NF-KB mediated by NF-KBinducing kinase (NIK) and IKB kinase (IKK) complex, which consists of IKK $\alpha$, IKK $\beta$, and NEMO. This is then continued with the activation of IKK $\alpha$ and IKK kinase. IKK actively phosphorylates IKB and causes ubiquitination and subsequent proteasomal degradation. IKB degradation translates NF-KB to the nucleus and acts as a transcription factor to induce the expression of anti-apoptotic and anti-inflammatory genes. Thus, if there is a failure in this pathway, apoptosis does not occur so that it triggers carcinogenesis [19].

The ability of cells to invade and carry out cell angiogenesis is also influenced by levels of matrix metalloproteinases (MMP), which facilitate the degradation and reorganization of the extracellular matrix (ECM). ROS regulates not only MMP expression but also TIMP (metalloproteinase tissue inhibitor) inactivation [19]. Oxidants also influence the release of inflammatory cytokines by innate immune receptors and the initiation of adaptive cellular response processes [20].

In conditions of obesity, inflammation is aggravated by an increase in macrophage infiltration and expression of proinflammatory cytokines. Various things can cause chronic inflammatory conditions, for example, oxidative stress, reduction of adiponectin, increased leptin, adipocyte death, macrophage infiltration, and lipolysis [7].

\section{DIABETES THERAPY AND CANCER RISK}

Diabetes drugs function as a substitute for insulin in the circulation and reduce hyperglycemia by different mechanisms. Metformin (1,1-dimethyl biguanide hydrochloride) is one of the most commonly prescribed diabetes drugs with a mechanism of decreasing liver gluconeogenesis and increasing skeletal muscle glucose absorption. In addition to lowering blood sugar and other DM complications, the drug is also known to reduce the incidence of cancer. The anti-proliferative effect of Metformin is mediated by suppression of the mTOR pathway and inactivation of IGF1R, both of which play an essential role in cell growth and are often dysregulated in cancer patients who have high levels of expression of this protein. Besides, metformin can also reversibly inhibit complex I of the mitochondrial respiratory chain and thus decreasing ROS production [21].

Metformin use is reported to be associated with a lower risk of colon, liver, pancreas, or breast cancer, and is not associated with prostate cancer risk [22-24]. A meta-analysis by Liu et al. [25] showed that both the risks of colorectal adenoma and colorectal cancer decreased significantly in diabetic patients using metformin. The colorectal cancer risk decreased by $22 \%$ in diabetic patients using metformin when compared to non-metformin users and other treatment users. 
Conversely, insulin users have a higher risk of developing cancer during their lifetime. Insulin use for more than or equal to 3 months without complications has a cancer risk that significantly increases by 2.4 times in the 10-12 years since the initial period of use. This is significantly related to cancer of the lung, liver, colon, pancreas, and bladder [26].

Pioglitazone, one of the anti-diabetics in the thiazolidinedione class, lowers blood glucose by enhancing peripheral insulin sensitivity via activation of peroxisome proliferator-activated receptor (PPAR) gamma. A randomized controlled trial study in mice showed that the administration of metformin (500 and $850 \mathrm{mg} / \mathrm{kg} /$ day) and pioglitazone (15 mg/kg/day) for 15 days resulted in a statistically significant reduction in pulmonary adenoma formation when compared to untreated controls, both as a single agent and in combination. A combination of metformin and pioglitazone performed is equal to metformin alone and better than pioglitazone at $15 \mathrm{mg} / \mathrm{kg} /$ day [27]. A randomized placebocontrolled phase-III trial showed that low dose metformin could reduce the prevalence and number of colorectal polyps after polypectomy in one year in non-diabetic patients with no side effects [28]. Another preliminary data on the overweight or obese postmenopausal women at increased risk for breast cancer suggests that metformin treatment has a direct effect on breast tissue of reducing pro-survival pathways, such as mTOR and TGF- $\beta$, and activating metabolic pathways, such as AMPK [29]. Despite the convincing results of the existing trials, more human clinical studies are needed to include antidiabetes therapy in cancer prevention guidelines.

\section{Recommendation}

Some recommendations were made to suppress the carcinogenic process that occurs in DM patients. Emphasizing lifestyle changes is suggested; some of these efforts include diet management, physical exercise, and blood glucose control. The primary goal in the diet management of DM is to achieve as near euglycemic response as possible. This can be done by doing carbohydrate counting and avoiding foods high in glycemic index and glycemic load, as the amount of carbohydrate consumed has the strongest influence on the glycemic response [30]. Carbohydrate sources that contain high fiber such as whole-grain bread and cereals, whole fruits, and vegetables are preferred in DM because fiber promotes satiety, can help reduce body weight, maybe less caloric, and also reduce colon cancer risk [31].

Recommended exercise in DM patients is a combination of aerobic, resistance, and flexibility exercises. Moderate to heavy intensity aerobic exercise is recommended at least 150 minutes per week with no two consecutive days or shorter duration (a minimum of 75 minutes per week) in young and physically fit individuals. Moderate to heavy intensity resistance exercise is recommended two to three times per week on non-consecutive days. In older people, flexibility exercises are recommended two to three times per week to increase muscle strength, balance, and flexibility. It is also recommended to reduce sedentary lifestyle, in which light activity every 30 minutes is proven to have an advantage in stabilizing blood sugar [32].

Nevertheless, it is advised to do pre-exercise health screening and consultation as it does carry some potential health risks, including acute complications such as cardiac events, hypoglycemia (especially in patients using insulin therapy), and hyperglycemia. One effort to reduce complications during exercise is to do continuous glucose monitoring (CGM), intending to be able to prevent and manage hypoglycemia earlier [33].

\section{REFERENCES}

1. Cho NH, Shaw JE, Karuranga S, Huang $Y$, RochaFernandes JD, Ohlrogge AW, et al. IDF Diabetes Atlas: Global estimates of diabetes prevalence for 2017 and projections for 2045. Diabetes Res and Clin Pract. 2018;138:271-81.

2. World Health Organization. Diabetes. Available from: https://www.who.int/news-room/fact-sheets/detail/ diabetes [Accessed 4 December 2019].

3. Ferlay J, Soerjomataram I, Dikshit R, Eser S, Mathers $\mathrm{C}$, Rebelo $\mathrm{M}$, et al. Cancer incidence and mortality worldwide: Sources, methods and major patterns in GLOBOCAN 2012. Int J Cancer. 2015;136:359-86.

4. Giovannucci E, Harlan DM, Archer MC. Diabetes and cancer: a consensus report. Diabetes Care. 2010;33(7):1674-85.

5. Arcidiacono B, liritano S, Nocera A, Possidente $K$, Nevolo $M$, Ventura $V$, et al. Insulin resistance and cancer risk: An overview of the pathogenetic mechanisms. Exp Diabetes Res. 2012;1:1-12.

6. Vázquez-Jiménez JG, Roura-Guiberna A, Jiménez-Mena $L R$, Olivares-Reyes JA. Role of free fatty acids on insulin resistance. Gac Med Mex. 2017;153:773-83.

7. Denduluri SK, Idowu O, Wang Z, Liao Z, Yan Z, Mohammed MK, Luu $\mathrm{HH}$. Insulin-like growth factor (IGF) signaling in tumorigenesis and the development of cancer drug resistance. Genes Dis. 2015;2(1):13-25.

8. Cifarelli V, Hursting SD. Obesity, diabetes, and cancer: A mechanistic perspective. Int J Diabetol Vasc Dis Res. 2015;4:1-14.

9. González N, Prieto I, Del Puerto-Nevado L. 2017 Update on the relationship between diabetes and colorectal cancer: epidemiology, potential molecular mechanisms and therapeutic implications. Oncotarget. 2017;8(11):18456-85. 
10. Calle E, Kaaks R. Overweight, obesity and cancer: epidemiological evidence and proposed mechanisms. Nat Rev Cancer. 2004;4(8):579-91.

11. Duan WX, Shen X, Lei JJ, Xu QH, Yu YT, Li R et al. Hyperglycemia, a neglected factor during cancer progression. BioMed Res Int. 2014;2014:1-10.

12. Pavlova NN, Thompson CB. The emerging hallmarks of cancer metabolism. Cell Metab. 2016;23:27-47.

13. Alisson-Silva F, Freire-de-Lima L, Donadio JL, Lucena $M C$, Penha L, Sa-Diniz JN, et al. Increase of O-glycosylated oncofetal fibronectin in high glucoseinduced epithelial-mesenchymal transition of cultured human epithelial cells. PLoS ONE. 2013;8:e60471.

14. Chocarro-Calvo A, García-Martínez JM, ArdilaGonzález S, De la Vieja A, García-Jiménez C. Glucoseinduced $\beta$-catenin acetylation enhances $W n$ signaling in cancer. Mol Cell. 2013;49:474-86.

15. White BD, Chien AJ, Dawson DW. Dysregulation of Wnt/ $\beta$-catenin signaling in gastrointestinal cancers. Gastroenterology. 2012;142(2):219-32.

16. Moore MM, Chua W, Charles KA, Clarke SJ. Inflammation and cancer: causes and consequences. Clin Pharmacol Ther. 2010;87:504-8.

17. Del-Prete A, Allavena P, Santoro G, Fumarulo R, Corsi MM, Mantovani A. Molecular pathways in cancerrelated inflammation. Biochem Med. 2011;21:26475.

18. Li Q, Engelhardt JF. Interleukin-1 beta induction of NF-kappaB is partially regulated by $\mathrm{H} 2 \mathrm{O} 2$-mediated activation of NF-kappaB-inducing kinase. J Biol Chem. 2006;281(3):1495-505.

19. Albensi BC. What is nuclear factor kappa B (NF-kB) doing in and to the mitochondrion? Front Cell Dev Biol. 2019;7:154.

20. Pitocco D, Zaccardi F, Di Stasio E, Romitelli F, Santini SA, Zuppi C, Ghirlanda G. Oxidative stress, nitric oxide, and diabetes. Rev Diabet Stud. 2010;7:15-25.

21. Ben Sahra, I. Metformin, independent of AMPK, induces mTOR inhibition and cell-cycle arrest through REDD1. Cancer Res. 2011;71:4366-72.

22. Currie CJ, Poole CD, Gale EA. The influence of glucose-lowering therapies on cancer risk in type 2 diabetes. Diabetologia. 2009;52:1766-77.

23. Hsieh MC, Lee TC, Cheng SM, Tu ST, Yen MH, Tseng $\mathrm{CH}$. The influence of type 2 diabetes and glucoselowering therapies on cancer risk in the Taiwanese. Exp Diabetes Res. 2012;41:1-6.
24. Mogavero A, Maiorana MV, Zanutto S, et al. Metformin transiently inhibits colorectal cancer cell proliferation as a result of either AMPK activation or increased ROS production. Sci Rep. 2017;7(1):1-9.

25. Liu F, Yan L, Wang Z, Lu Y, Chu Y, Li X, et al. Metformin therapy and risk of colorectal adenomas and colorectal cancer in type 2 diabetes mellitus patients: A systematic review and meta-analysis. Oncotarget. 2017;8:16017-26.

26. Cho O, Oh YT, Chun M, Noh OK, Heo J. Influence of insulin therapy for type 2 diabetes mellitus on cancer incidence. J Clin Oncol 2017;35:15_suppl, e13036-e13036.

27. Seabloom DE, Galbraith AR, Haynes AM, Antonides JD, Wuertz BR, Miller WA. Fized-dose combinations of Pioglitazone and Metformin for lung cancer prevention. Cancer Prev Res (Phila). 2017;10(2):116-23.

28. Higurashi T, Hosono K, Takahashi H, Komiya Y, Umezawa S, Sakai E, et al. Metformin for chemoprevention of metachronous colorectal adenoma or polyps in post-polypectomy patients without diabetes: a multicentre double-blind, placebo-controlled, randomised phase 3 trial. The Lancet Oncology. 2016;17:475-83.

29. Ballinger T, Marino N, German R, True J, Lu X, Cao $S$, et al. Prospective, placebo-controlled, randomized study of metformin for breast cancer prevention in overweight/ obese women. Cancer Res. 2020;80: P113-03.

30. American Diabetes Association. Diabetes Care. 2019;42 Supplement 1:S46-S60

31. Dahl WJ, Stewart ML. Position of the academy of nutrition and dietetics: Health implications of dietary fiber. J Acad Nutr Diet. 2015;115(11):1861-70.

32. Colberg SR, Sigal RJ, Yardley JE, Riddell MC, Dunstan DW, Dempsey PC, et al. Physical Activity/Exercise and Diabetes: A Position Statement of the American Diabetes Association. Diabetes Care. 2016. 39(11):2065-79.

33. Riddell MC, Milliken J. Preventing exercise- induced hypoglycemia in type 1 diabetes using real-time continuous glucose monitoring and a new carbohydrate intake algorithm: an observational field study. Diabetes Technol Ther. 2011;13:819-25. 